\title{
Electromagnetic Techniques to Minimize the Risk of Hazardous Local Heating around Medical Implant Electrodes during MRI Scanning
}

\author{
Steven McCabe \& Jonathan Scott \\ Department of Engineering \\ The University of Waikato \\ Hamilton, New Zealand \\ Email: som1@ @students.waikato.ac.nz
}

\author{
Stephen Butler \\ Midland MRI \\ Hamilton, New Zealand
}

\begin{abstract}
Magnetic Resonance Imaging (MRI) scans are contraindicated for many patients with medical implants. We establish the circumstances that cause, and the resistances required to ameliorate and to eliminate dangerous levels of MRI-induced heating that occur at the exposed, distal end of an electrical lead implanted in tissue. Simulated predictions are compared with measurements made at $128 \mathrm{MHz}$ in a 3-Tesla MRI machine. A low resistance at kilohertz frequencies is sought by implant makers, in contrast with the high resistance demanded for safety. The practicality of presently-developed strategies to prevent tissue damage is brought into question. We examine the extent to which skin-depth and transmission-line properties can be manipulated to improve safety.
\end{abstract}

Index Terms-Biomedical electrodes, medical diagnostic imaging, dipole antennas, electromagnetic modeling.

\section{INTRODUCTION}

The strong magnetic field present in a Magnetic Resonance Imaging (MRI) machine presents a hazard, as most people know. Nevertheless, it is the energy delivered by the highpower RF field that is of greater concern for the patient. A 3Tesla (3 T) MRI machine can deliver peak pulses in the order of $30 \mathrm{~kW}$ at $128 \mathrm{MHz}$. [1] This can lead to significant heating of the patient. The International Commission on Non-Ionizing Radiation Protection (ICNIRP) recommends maximum localized temperatures of $38^{\circ} \mathrm{C}$ in the head and $39^{\circ} \mathrm{C}$ in the torso, representing $1-2{ }^{\circ} \mathrm{C}$ of heating. [2] The dielectric heating of a patient is monitored by the Specific Absorption Rate (SAR), the power absorption per unit mass. The SAR is typically averaged over the whole-body, whole-head, or $10 \mathrm{~g}$ of mass.

The leads associated with medical implants can behave as antennas and concentrate the RF field. [3] We will show that this phenomenon is worst when the lead is just short of one-half wavelength long, about $250 \mathrm{~mm}$ for a typical lead in standard saline in a $3 \mathrm{~T}$ machine. There are claims in the literature that $10 \mathrm{~g}$ is too coarse near implants [4], and we confirm and exemplify this. While pacemakers have short leads, Spinal-Cord Stimulation (SCS) and Deep-Brain Stimulation (DBS) systems have leads that can exceed $600 \mathrm{~mm}$ in length because the implant is situated in the abdomen or chest cavity, far from the distal end of the electrode.

The MRI safety of implants has become a global issue and sites such as "Shellock" [5] are consulted throughout the world by MRI radiologists. Many implant-wearers are contraindicated for MRI scans. There is a growing need to address this problem. A spate of patents appeared in the last decade supposedly addressing the issue [6]-[11]. Only one product has appeared and it is rated for use only in $1.5 \mathrm{~T}$ machines, and only with a restricted scanning protocol. [12]

\section{IMPACT OF A BARE WIRE}

An implant lead wire acts as an "antenna in saline". In a lossless medium (conductivity $\sigma=0$ ), an ordinary dipole antenna will resonate when excited by an RF field when its length is approximately equal to an odd integer multiple of the half-wavelength: $0.5 \lambda, 1.5 \lambda, 2.5 \lambda, \cdots$. In the conductive saline medium, the wave will compress and the wavelength becomes [13]:

$$
\lambda=\sqrt{\frac{8 \pi^{2}}{\omega^{2} \mu \epsilon}} \cdot\left[\sqrt{1+\left(\frac{\sigma}{\omega \epsilon}\right)^{2}}+1\right]^{-1 / 2}
$$

where $\omega$ is the angular frequency, and $\mu$ and $\epsilon$ are the permeability and permittivity of the medium, respectively. Furthermore, the induced antenna currents are not confined by the ends of the wire and are free to extend into the medium. [14] The antenna will be effectively lengthened by the medium itself.

This situation leads us to expect heating to occur at the ends of a wire when stimulated at a frequency slightly below that at which the lead wire is one-half wavelength long. To confirm this expectation we simulate using COMSOL Multiphysics 4.4 with a phantom model set according to ASTM F2182-11a [15] with the exception of the length parameter, which is doubled from $0.6 \mathrm{~m}$ to $1.2 \mathrm{~m}$, to accommodate the testing of longer length wires. For simulation details refer to [16].

Fig. 1 shows the simulated field at the end of a bare wire embedded in a human-body phantom and exposed to a calibrated SAR of $1 \mathrm{~W} / \mathrm{kg}$ against length normalized to the wavelength via (1). Simulation confirms a strong resonance at $0.41 \lambda_{P}$, confirming theoretical expectation.

\section{IMPACT OF INSUlated Wire With Distal END EXPOSED}

Now we consider a wire with insulation covering all but one end, representing an implant's distal electrode. The average electric field near the distal electrode at various wire lengths is in Fig. 2. The length is normalized to $\lambda_{P n}=0.61 \mathrm{~m}$ and the first resonant peak occurs at $0.41 \lambda_{P n}$. The insulation shifted 


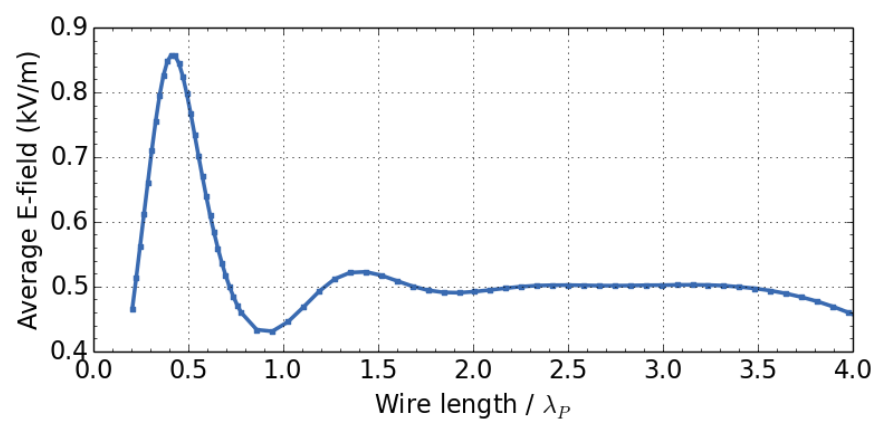

Fig. 1. Average E-field at one end of a $800 \mu \mathrm{m}$ diameter bare wire for various lengths in a lossy phantom $(\sigma=0.47 \mathrm{~S} / \mathrm{m})$ to model a human body. The wire length is normalized to a wavelength of $0.24 \mathrm{~m}$.

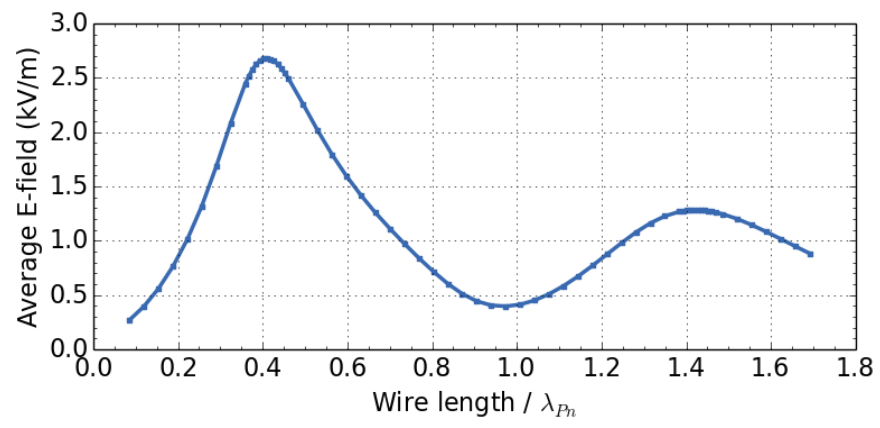

Fig. 2. Average E-field at the distal electrode of an insulated wire in a lossy phantom $(\sigma=0.47 \mathrm{~S} / \mathrm{m})$. The insulation covering the $800 \mu \mathrm{m}$ diameter wire is $350 \mu \mathrm{m}$ thick. The wire length is normalized to a wavelength of $0.61 \mathrm{~m}$.

the resonant frequency and it has also increased the electric field intensity at the first resonant peak threefold.

The change in temperature $\left(T-37^{\circ} \mathrm{C}\right)$ at the distal electrode of the insulated wire after 5 min of MRI scanning is considered next. The result with and without blood perfusion is shown in Fig. 3, again with the $x$-axis normalized to the wavelength of the electric field within the phantom, $\lambda_{P n}$.

The inclusion of blood perfusion provides an approximation to living human tissue, reducing the heating by $18 \%$ at the worst-case length. At $0.41 \lambda_{P n}(0.25 \mathrm{~m})$, normal body temperature is exceeded by almost $22{ }^{\circ} \mathrm{C}$, well over the $2{ }^{\circ} \mathrm{C}$ limit for SCS. The peak temperature declines at longer wire lengths but still mostly exceeds safety limits. A two-dimensional slice of the temperature $T$ in the $x-z$ plane at $y=0$ is shown in Fig. 4, for a wire length of $0.41 \lambda_{P n}$. This figure makes it clear that a sample size of $10 \mathrm{~g}$ is indeed too coarse for estimating $\mathrm{SAR}-10 \mathrm{~g}$ is a sphere of one inch diameter, large compared to the hot region of the plot.

\section{Comparison with Measured Values}

In order to have confidence in our simulations, a torso-andhead phantom after [15] was built in clear acrylic and filled with $28 \mathrm{~L}$ of saline gel with a conductivity of $0.47 \mathrm{~S} / \mathrm{m}$. The ratio of $\mathrm{NaCl}$ and polyacrylic acid (PAA) to distilled water was $1.32 \mathrm{~g} / \mathrm{L}$ and $10 \mathrm{~g} / \mathrm{L}$, respectively. Wire samples in the phantom were tested within a commercial 3T MRI scanner. Fig. 5 shows the phantom on the MRI machine bed.

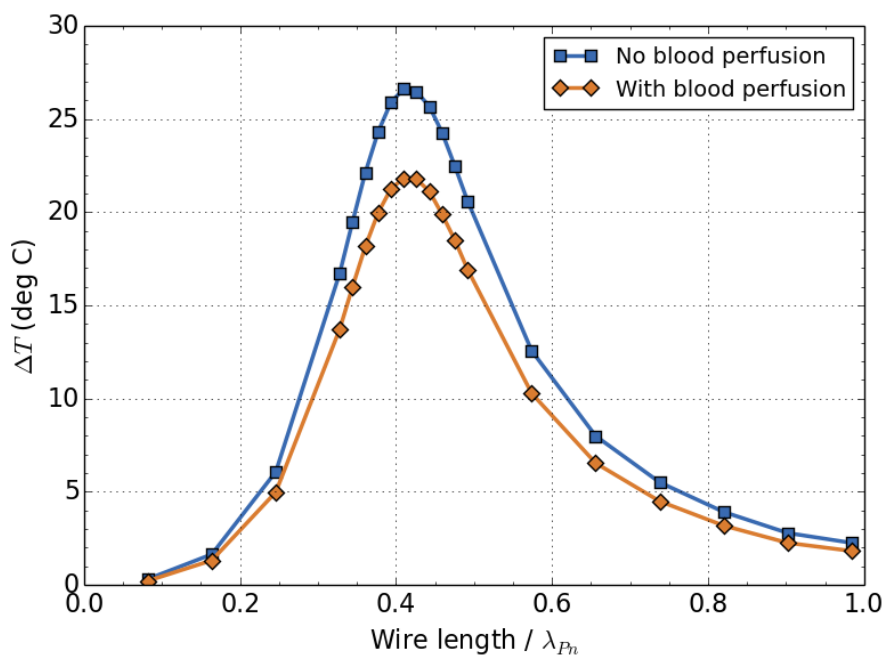

Fig. 3. The change in temperature $\Delta T$ near the distal electrode of the insulated wire after 5 minutes of simulated scanning. The insulation covering the $800 \mu \mathrm{m}$ diameter wire is $350 \mu \mathrm{m}$ thick. The length of the wire is normalized to a wavelength of $0.61 \mathrm{~m}$.

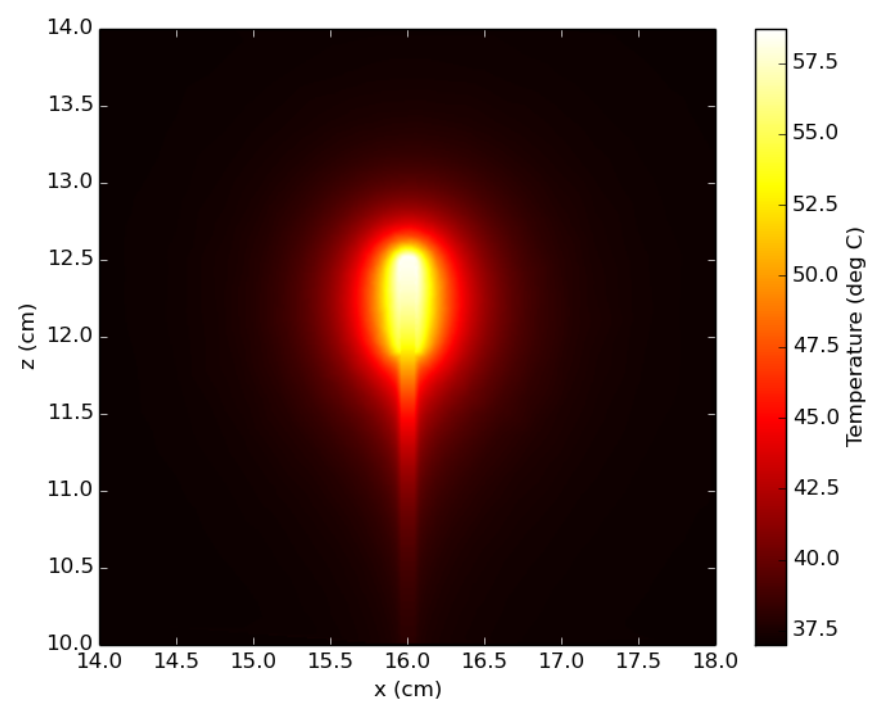

Fig. 4. The worst-case $T(x, z)$ at the distal electrode after 5 minutes of simulated scanning. The wire length was $0.41 \lambda_{P n}$ and blood perfusion was included. The initial temperature was $37^{\circ} \mathrm{C}$.

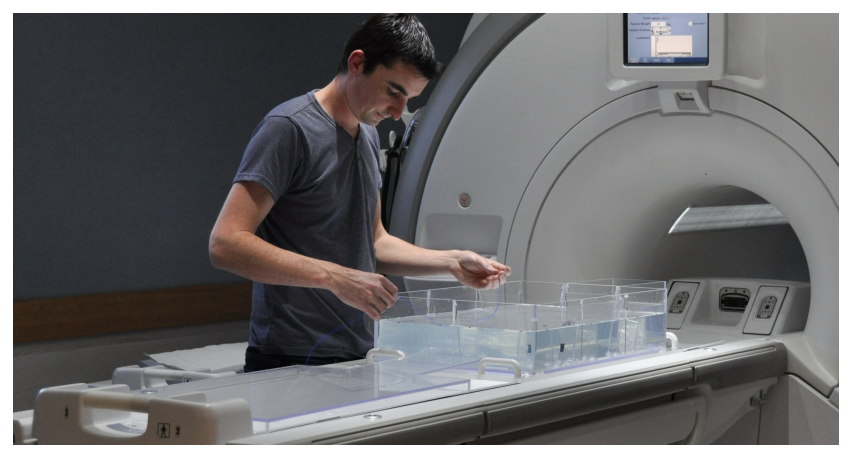

Fig. 5. Clear acrylic phantom on the bed of the MRI machine with operator. 


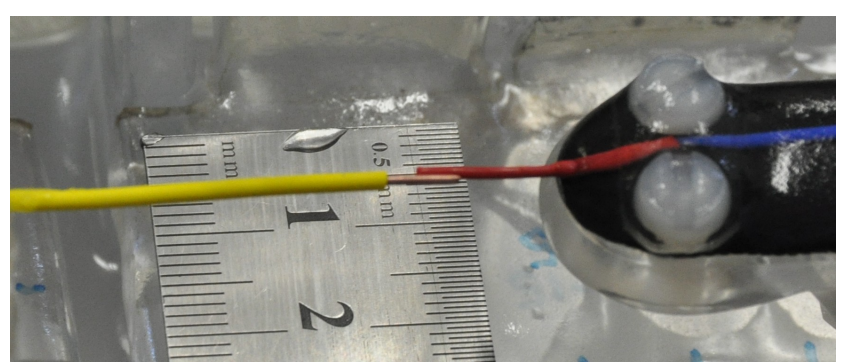

Fig. 6. Photograph of the Optocon TS2 probe (red) aligned alongside the bared end of the insulated wire sample (yellow). Scale is in centimeters. An adjustable mechanism supports the TS2 probe and holds it in position.

Temperature was measured using an Optocon Fotemp GaAs-based fiber-optic system with TS2 probes. Fig. 6 depicts a temperature sensor tip aligned with the exposed tip of an $800 \mu \mathrm{m} \mathrm{Cu}$ wire of length $0.41 \lambda$, coated with $350 \mu \mathrm{m}$ of plastic insulation. 1

The temperature rise after 5 minutes of MRI scanning was recorded for various lengths of insulated wire. The insulation was $350 \mu \mathrm{m}$ thick, but thinner and thicker variants were also tested. The measurements are shown in Fig. 7 with simulated data overlaid. We measure a temperature rise of $7.9^{\circ} \mathrm{C}$ near the worst-case length, with an estimated whole-body SAR of $2.7 \mathrm{~W} / \mathrm{kg}$. We scale our measurements by 2.7 times to account for the conservative SAR estimate reported by the MRI machine. This corresponds to a rise of $21.3^{\circ} \mathrm{C}$ for a whole-body SAR of $1 \mathrm{~W} / \mathrm{kg}$. Similar wire with double the thickness of insulation produces almost $25.7^{\circ} \mathrm{C}$, while just $21 \mu \mathrm{m}$ of insulation produces only $3.7^{\circ} \mathrm{C}$, consistent with our expectations in section VII.

\section{IMPACT OF WIRE RESISTANCE}

Implant manufacturers seek a low resistance connection to the electrodes to minimize the voltage required to deliver therapeutic current and maximize battery life. An SCS implant typically delivers a maximum current of $10 \mathrm{~mA}$. A resistance of $50 \Omega / \mathrm{m}$ is desirable, double that barely comfortable.

A reduction in the heating of the tissue near the distal electrode could be achieved with uniformly distributed resistance in the implant wire. Three-dimensional simulations of a hollowed out version of the implant wire with $350 \mu \mathrm{m}$ of insulation were performed. The resistance was varied by controlling the resistivity of the metal. To ensure current uniformity, the resistivity was constrained to values such that the skin depth exceeded the thickness of the metal by at least 10 times. The resistance at $128 \mathrm{MHz}$ and the temperature change after $5 \mathrm{~min}$ as a function of the metal resistivity is shown in Fig. 8. A $2{ }^{\circ} \mathrm{C}$ limit would require $>850 \Omega / \mathrm{m}$ of resistance. The significant benefit of a factor of two would require $>200 \Omega / \mathrm{m}$.

\footnotetext{
${ }^{1}$ Optocon say that the "final few millimeters" of the probe is the active sensing region. Our experience suggests that the temperature of the last $1 \mathrm{~mm}$ of the probe tip contributes to the reading. We have chosen to align the probe and sample wire, as shown in figure 6 , such that the sensitivity is optimised.
}

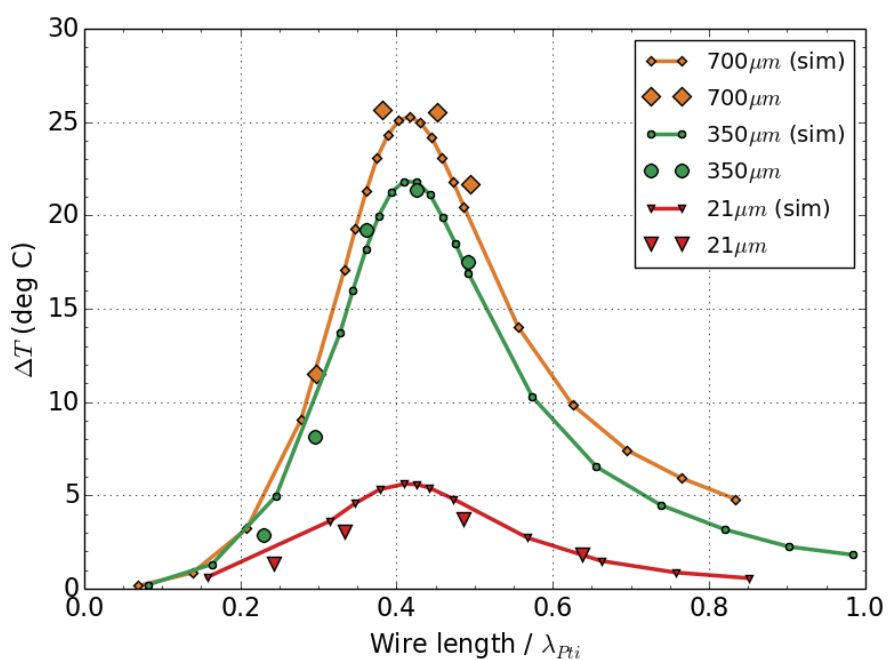

Fig. 7. The change in temperature $\Delta T$ near the distal electrode for three types of insulated wires, varying by insulation thickness, after 5 minutes scanning. Simulated data is verified by several measurements about the resonant peaks. The $x$-axis is normalized individually for each insulation type, such that the peaks occur at $0.41 \lambda_{P t i}$. The respective wavelengths are $\lambda_{P 700}=0.72 \mathrm{~m}$, $\lambda_{P 350}=0.61 \mathrm{~m}$, and $\lambda_{P 21}=0.32 \mathrm{~m}$.

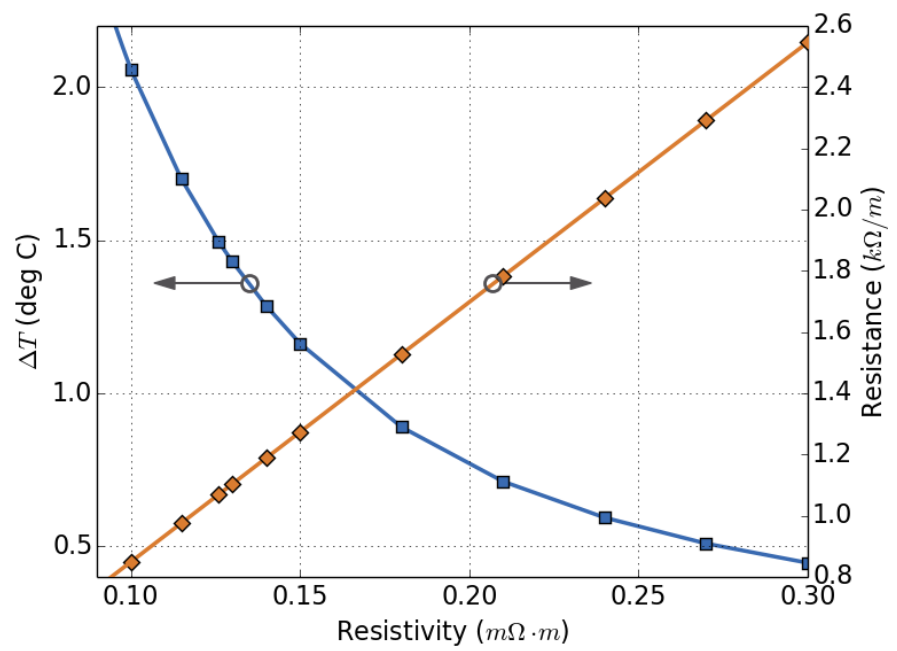

Fig. 8. The change in temperature $\Delta T$ near the distal electrode after $5 \mathrm{~min}$ of simulated scanning for various amounts of uniform wire resistance. The insulation is $350 \mu \mathrm{m}$ thick. The linearity between the resistance and resistivity confirms that the skin depth is sufficiently larger than the metal thickness, such that the current is uniform.

\section{IMPACT OF SKIN EFFECT}

Is it possible to select values for the radius $r$ and resistivity $\rho$ of the wire such that the skin effect provides high enough resistance at $128 \mathrm{MHz}$ and low enough at dc? Skin depth $\delta \approx \sqrt{2 \rho / \omega \mu}$ [17], about $14.5 \mu \mathrm{m}$ for Pt. Resistance of a wire $R=\rho / A \Omega / \mathrm{m}$, where $A$ is effective cross-sectional area. For dc, $A=\pi r^{2}$, but smaller for increasing frequency, and approximated as $2 \pi r \delta$ if $r \gg \delta$. It is clear that dc resistance is inversely proportional to $r^{2}$ and to first order ac resistance to $r$. Thus larger $r$ will give a larger ratio of ac to dc resistance, but falling total resistance. To have no more than $R_{d c}=50 \Omega / \mathrm{m}$ in Pt requires $r \gtrsim 26 \mu \mathrm{m}$. Simple approximations for the 


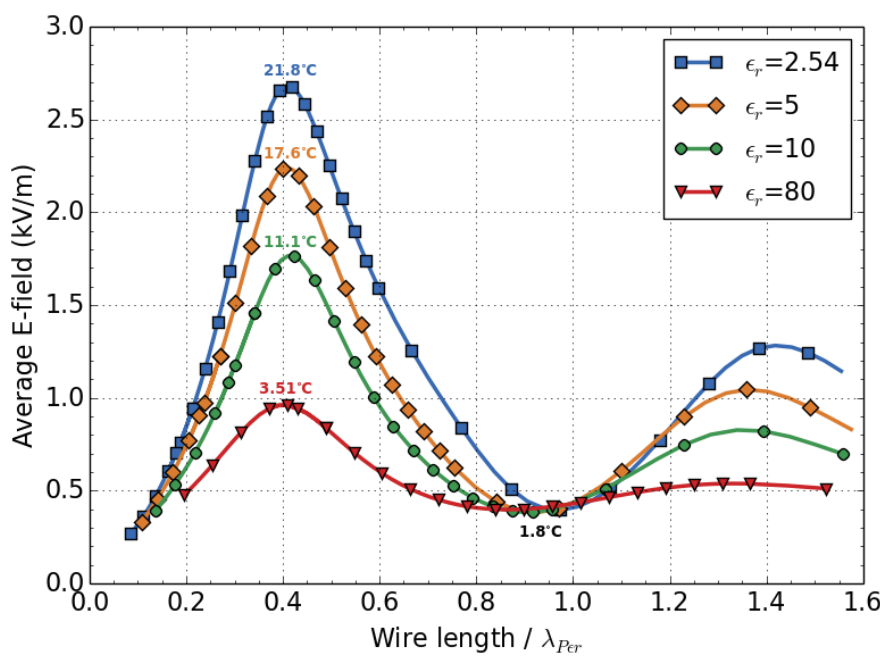

Fig. 9. Average E-field at the distal electrode for various insulation permittivities when $t_{i}=350 \mu \mathrm{m}$. Several values for $\Delta T$ (simulated) are overlaid. The $x$-axis is normalized individually for the different traces.

effective area fail since $r$ is comparable to $\delta$, but the Modified Lorentzian Method of [18] yields $R_{a c}=59 \Omega / \mathrm{m}$. Results are the same for all metals (ratio does not scale with $\rho$ ). Long before ac exceeds dc resistance sufficiently, both become quite small. [16] Skin depth may help, but it cannot deliver the required ratio.

\section{IMPACT OF INSULATION PROPERTIES}

Varying the thickness, $t_{i}$, and the dielectric constant, $\epsilon_{r}$, of the insulation leads to the results shown in Fig. 7 and Fig. 9. These results can be understood by realizing that an implanted lead wire resembles a coaxial transmission line but with conductive tissue representing the outer cylindrical conductor. [19] The ideal characteristic impedance of a uniform coaxial transmission line is

$$
Z_{0}=\frac{1}{2 \pi} \sqrt{\frac{\mu}{\epsilon}} \cdot \ln \left(\frac{r_{2}}{r_{1}}\right)
$$

where $r_{1}$ and $r_{2}$ are the radii of the inner and outer conductors, and $\mu$ and $\epsilon$ are the absolute permeability and permittivity of the dielectric material, respectively. Its $Z_{0}$ affects the amount of RF energy that is coupled to the implant lead wire. It is clear that thin insulation, while potentially not practical for reasons of durability, offers a significant advantage. An insulation with a high dielectric constant will also help.

\section{CONCLUSION}

We explain the mechanism behind the hazard presented by implant electrodes, and confirm that $10 \mathrm{~g}$ is too coarse a sample over which to average exposure. Simulated and measured results agree. We have established lead resistance values required to reduce or eliminate the heating hazard at $128 \mathrm{MHz}$, and to preserve SCS implant battery life for low-frequency signals. We show that the skin effect alone is unable to provide the impedance difference. We show that manipulation of the insulation properties can lower peak distal heating by a large extent. In combination with other techniques that we are not free to disclose at this time [20], can be expected to lead to an MRI-safe electrode design.

\section{ACKNOWLEDGEMENT}

We would like to thank the Waikato Medical Research Foundation (WMRF) for funding. We also acknowledge WaikatoLink and KiwiNet for their valuable support.

\section{REFERENCES}

[1] R. Caverly, G. Breed, W. Cantrell, M. Eron, J. Garcia, N. Kondrath D. Myer, M. Ruiz, and J. Walker, "Advancements at the lower end: Advances in HF, VHF, and UHF systems and technology", IEEE Microwave Magazine, vol.16, no.1, pp.28-49, Feb. 2015.

[2] "ICNIRP statement on: Medical magnetic resonance (MR) procedures: Protection of patients", International Commission on Non-Ionizing Radiation Protection (ICNIRP), Health Phys., vol. 87 no. 2, pp. 197-216, Aug. 2004.

[3] J. A. Nyenhuis, P. Sung-Min, R. Kamondetdacha, A. Amjad, F. G. Shellock and A. R. Rezai "MRI and implanted medical devices: Basic interactions with an emphasis on heating", IEEE Trans. Dev. Mat. Rel., vol.5, no.3, pp.467-480, Sept. 2005.

[4] L. M. Angelone, J. Ahveninen, J. W. Belliveau and G. Bonmassar, "Analysis of the role of lead resistivity in specific absorption rate for deep brain stimulator leads at 3T MRI", IEEE Trans. Med. Imag., vol.29, no.4, pp.1029-1038, Apr. 2010.

[5] "MRISAFETY.COM, Your information resource for MRI safety, bioeffects, \& patient management", http://www.mrisafety.com/ retrieved Sept 2014.

[6] J. M. Olsen, S. L. Bolea, G. A. Hrdlicka, C. D. Wahlstrand, and T. B. Hoegh, "Lead electrode for use in an MRI-safe implantable medical device", International patent number WO 2006/093685 A1, Sept, 2006.

[7] C. D. Wahlstrand and T. B. Hoegh, "Electrode for use in an MRI-safe implantable medical device", European patent number EP 1742701 B1, Aug, 2010.

[8] C. D. Wahlstrand, G. A. Hrdlicka, R. M. Skime, P. Przybszewski, and T. E. Cross, "Energy shunt for producing an MRI-safe implantable medical device", International patent number WO 2005/030322 A1, Apr, 2005.

[9] B. A. Tranchina, "Method for fabricating a stimulation lead to reduce MRI heating", US patent number 8,601,672 B2, Dec, 2013.

[10] G. Bonmassar and E. Eskandar, "MRI compatible leads for a deep brain stimulation system", International patent number WO2014055737 A1, Apr, 2014.

[11] T. B. Hoegh, S. L. Bolea, C. D. Wahlstrand, G. A. Hrdlicka, and J. M. Olsen, "Lead electrode for use in an MRI-safe implantable medical device", European patent number EP 1740260 B1, Aug, 2010.

[12] MRI and SCS, SureScan system Medtronic, USA, Implant Manual, 2013. http://www.mrisurescan.com/au/for-radiologists/spinal-cord-stimulators/ mri-and-scs/index.htm\#tab2 retrieved Aug 2014.

[13] R. K. Shevgaonkar, Electromagnetic waves, Tata McGraw-Hill, pp. 175, 2006.

[14] J. E. Brown, "Radiofrequency heating near medical devices in magnetic resonance imaging", $\mathrm{PhD}$ dissertation, Univ. of Florida, May 2012.

[15] F2182-11a Standard test method for measurement of radio frequency induced heating on or near passive implants during magnetic resonance imaging, ASTM International, West Conshohocken, PA, USA, 2010.

[16] S. McCabe and J. Scott, "Cause and amelioration of MRI-induced heating through medical implant lead wires," Proceedings of the 21st Electronics New Zealand Conference, Hamilton, 20-21 Nov. 2014, pp3440.

[17] S. Ramo, J. R. Whinnery and T. Van Duzer, Fields and waves in communication electronics, second edition, John Wiley \& Sons, Inc, pp. 147-150, 1984.

[18] D. W. Knight, "Practical continuous functions for the internal impedance of solid cylindrical conductors", Apr. 2013.

[19] R. W. P. King and G. S. Smith, Antennas in matter: fundamentals, theory, and applications, The MIT Press, Cambridge, MA, 1981.

[20] J. B. Scott and S. O. McCabe, "Implant conductor with improved radio frequency properties", New Zealand provisional patent 708633, Filed on May 29, 2015. 